\title{
Analysis of Modeling and Vibration Performance of a New Type of Crop- ping Machine
}

\author{
Lijun Zhang ${ }^{1, *}$ and Shengdun Zhao ${ }^{2}$
}

${ }^{I}$ College of Electromechanical Engineering, China University of Petroleum, Qingdao, China, 266580; ${ }^{2}$ School of Me-
chanical Engineering, Xi'an Jiaotong University, Xi'an, China, 710049

\begin{abstract}
In order to reduce the cropping force and enhance the cropping cross-section quality, a new type of cropping machine is proposed in this paper. The basic structure and working principle of the cropping machine are introduced. Based on D'Alembert's principle, the vibration equations of the cropping machine along the $\mathrm{Y}$ direction, the $\mathrm{X}$ direction and around $\mathrm{X}, \mathrm{Y}, \mathrm{Z}$ axes are built in detail and the motion curves of the vibration body in all directions are also obtained. The amplitude frequency response characteristic curve of the vibration body in the Y direction is given and the resonance frequency of the vibration body in the $\mathrm{Y}$ direction is about $7.94 \mathrm{rad} / \mathrm{s}$. The cropping experimental results show that the cropping force acted on the metal bar is low and the cross-section quality is very high.
\end{abstract}

Keywords: Precision cropping, Vibration mechanism, Amplitude frequency response, Excited frequency.

\section{INTRODUCTION}

Cropping is often the first step of forging industry, and is also a key working procedure of producing the parts, such as standard pin, chain connecting pin, bearing roller and so on $[1,2]$. The cropping method in industry is mainly to shear the bar directly with a common die, and this process is plagued with a big shearing force, a bad cross-section, a short lifetime die and noise [3, 4]. Nowadays, a kind of lowstress manufacturing system appears. In the cropping process, V-shaped grooves are cut at specific length intervals in the metal bar to engender the stress concentration at the tip of the V-shaped groove. Then the slotted bar is fed to crop under the action of the fatigue loading and would rupture after certain periods. Haixia Li et al. carried out a large number of meaningful works by this low-stress cropping method and obtained the fracture morphology of 45 steel bars with a diameter of $30 \mathrm{~mm}$ [5]. Yong Tang et al. developed a lowstress rotary forging blanking machine and performed the cropping experimental study [6, 7]. However, the above methods need more cropping time than the existing shear methods for a segment of metal bar, which cannot meet the needs of industrialization.

Therefore, a new type of low-stress cropping system is proposed in this paper. Besides the use of the stress concentration effect of the groove, the cropping machine can produce the resonance effect in the initiation stage of crack of $\mathrm{V}$-shaped notch tip to further reduce the cropping time. The structure of the cropping machine is designed and its vibration performance is also analyzed in detail. The cropping experiments for 45 steel bars and 20 steel bars are carried out by means of the cropping machine.

*Address correspondence to this author at the College of Electromechanical Engineering, China University of Petroleum, Qingdao, China, 266580; Tel: 086-0532-86983500; E-mail: Zlj-2@163.com

\section{THE BASIC STRUCTURE AND WORKING PRIN- CIPLE OF THE CROPPING MACHINE}

The new type of low-stress cropping machine mainly consists of the electric machine, the eccentric block and the vibration body as shown in Fig. (1). The structure of the eccentric block is shown in Fig. (2). The vibration body mainly includes the bed plate, the kinetic die, the vibration spring and the kinetic pressure ring. When the cropping machine works, firstly the electric machine drives the eccentric block to rotate at the natural frequency of the vibrating body, which can produce the resonance effect in the initiation stage of crack to further reduce the initiation time of crack. Then the exciting force will act on the metal bar circularly and the crack at the tip of V-shaped groove further expands slowly and regularly. Finally the metal bar would rupture after certain periods. In order to sustain the vibration effect, there are 8 springs to support the vibration body.

\section{THE VIBRATION PERFORMANCE ANALYSIS OF THE CROPPING MACHINE}

\subsection{The Vibration Performance of the Cropping Ma- chine}

As is shown in Fig. (1), the cropping machine proposed in this paper is a kind of single axis inertia vibration machine. The exciting force caused by the two eccentric blocks does not pass through the center of mass of the vibration body, thus the vibration body will vibrate around its centroid. Based on the structure as shown in Fig. (1), the vibration mechanics model of the cropping machine is shown in Fig. (3). There are 5 degrees of freedom in the vibration cropping mechanism.

Suppose that the total mass supported by the springs is the mass $m$, which does not include the mass of two eccentric blocks. According to D'Alembert's principle [8], the forces acted on the vibration body, which are the inertia 


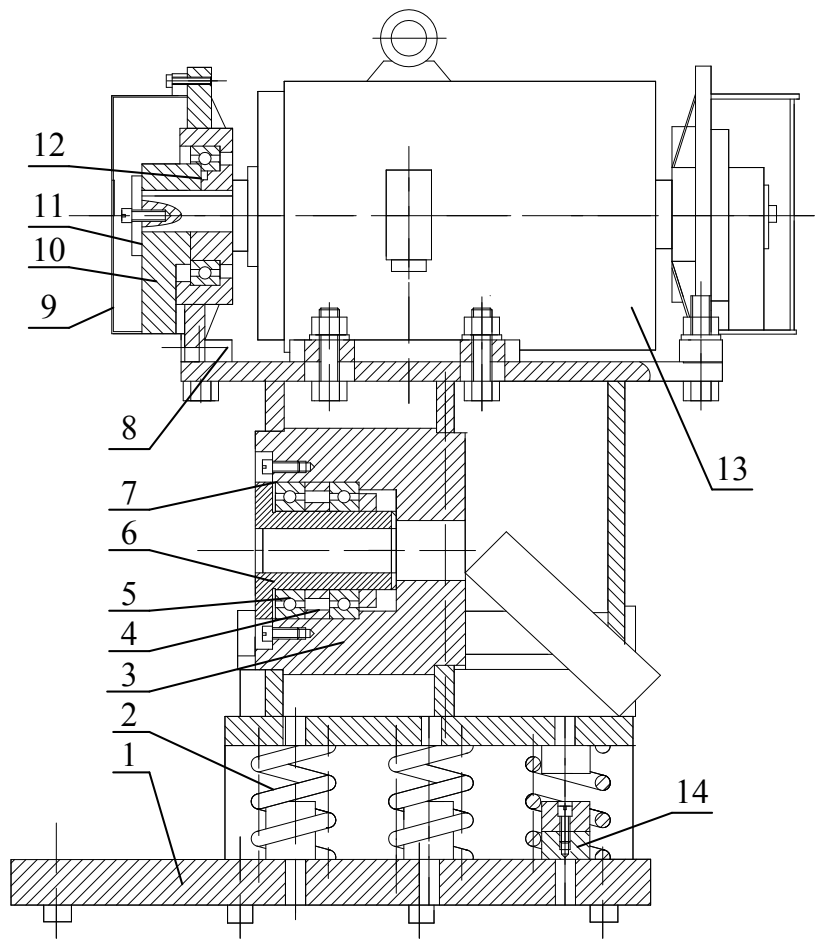

Fig. (1). The basic structure of the cropping machine. 1. bed plate 2 . vibration spring 3 . vibration grid 4 . outer sleeve 5 .inner sleeve 6 . kinetic die 7. kinetic pressure ring 8. supporting block 9. swing block protective sleeve 10 . eccentric block 11 . baffle plate 12 . swing block inside covering 13. electric machine 14. spring seat.

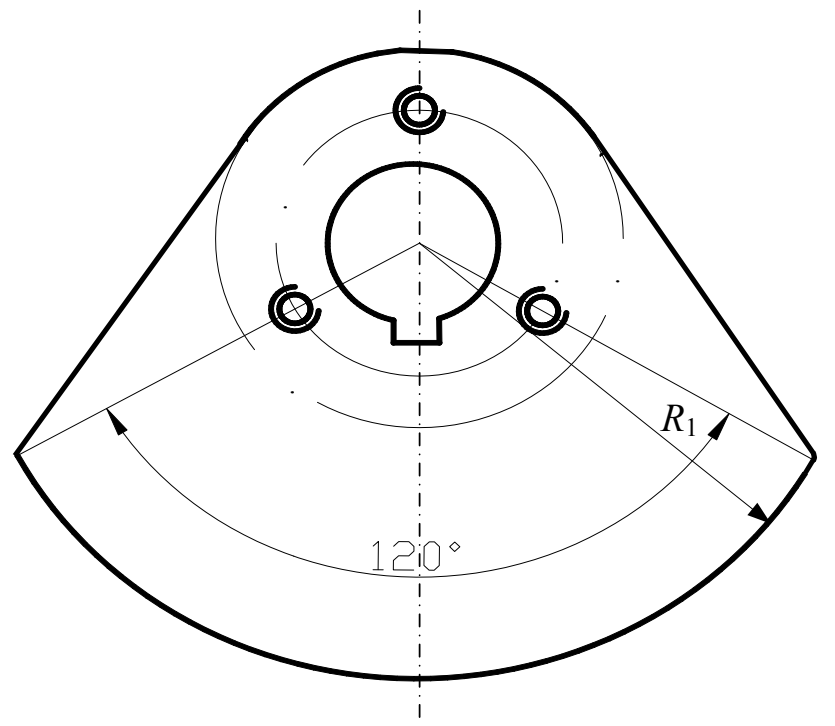

Fig. (2). The eccentric block.

force, the damping force, the elastic force, the exciting force and torque, caused by the exciting force should be balanceable and their resultant force is zero. Based on this, the vibration equations of the vibration body along the $\mathrm{Y}$ direction, the $\mathrm{X}$ direction and around the $\mathrm{X}$ axis, the $\mathrm{Y}$ axis, and the $\mathrm{Z}$ axis are given by [3]:

$$
\left\{\begin{array}{l}
\left(m+2 m_{0}\right) \ddot{\mathrm{y}}+k \dot{\mathrm{y}}=2 m_{0} \omega^{2} r \sin \omega t \\
\left(m+2 m_{0}\right) \ddot{\mathrm{x}}=2 m_{0} \omega^{2} r \cos \omega t \\
\left(J_{2}+J_{\mathrm{y}}\right) \ddot{\phi}_{\mathrm{y}}=m_{0} \omega^{2} r\left(L_{o z_{1}}-L_{o z_{2}}\right) \cos \omega \mathrm{t} \\
\left(J_{1}+J_{\mathrm{x}}\right) \ddot{\phi}_{\mathrm{x}}=m_{0} \omega^{2} r\left(L_{o z_{1}}-L_{o z_{2}}\right) \sin \omega t \\
\left(J_{3}+J_{\mathrm{z}}\right) \ddot{\phi}_{\mathrm{z}}=2 m_{0} \omega^{2} r L_{\text {oy }} \cos \omega t
\end{array}\right.
$$

where $J_{1}, J_{2}, J_{3}$ are the inertia moments of the vibration body along the $\mathrm{X}$ axis, the $\mathrm{Y}$ axis and the $\mathrm{Z}$ axis respectively; $J_{x}$, $J_{y}, J_{z}$ are the inertia moments of the eccentric block around the $\mathrm{X}$ axis, the $\mathrm{Y}$ axis and the $\mathrm{Z}$ axis respectively; $L_{\mathrm{oz} 1}, L_{\mathrm{oz} 2}$ are the distances between the force points of the two eccentric blocks and the center of mass of the vibration body along the $\mathrm{Z}$ direction respectively; $L_{o y}$ is the distance between the gyration center of two eccentric blocks and the center of mass of the vibration body along the $\mathrm{Z}$ direction; $m_{0}$ is the mass of the eccentric block; $w, r$ are the angular velocity of the electric machine shaft and the eccentric distance of the eccentric block respectively; $\ddot{\mathbf{x}}, \ddot{\mathbf{y}}$ are the accelerated velocities of the vibration body in the $\mathrm{X}$ direction and the $\mathrm{Y}$ direction respectively; $\ddot{\varphi}_{\mathrm{x}}, \ddot{\varphi}_{\mathrm{y}}, \ddot{\varphi}_{\mathrm{Z}}$ are the angular accelerations of the vibration body around the $\mathrm{X}$ axis, the $\mathrm{Y}$ axis and the $\mathrm{Z}$ axis.

The specific solutions to differential equations (1) are expressed as:

$\left\{\begin{array}{l}\mathbf{y}_{\mathbf{0}}=\lambda_{y} \sin \omega t \\ \mathbf{x}_{0}=\lambda_{y} \cos \omega t \\ \phi_{x}=\lambda_{\phi x} \cos \omega t \\ \phi_{y}=\lambda_{\phi y} \sin \omega t \\ \phi_{z}=\lambda_{\phi z} \cos \omega t\end{array}\right.$

where $\lambda_{x}, \lambda_{y}$ are the amplitudes of vibration caused by the exciting force in the $\mathrm{X}$ direction and the $\mathrm{Y}$ direction respectively; $\lambda_{\varphi x}, \lambda_{\varphi y}, \lambda_{\varphi z}$ are the arguments caused by the excited moments in the $\mathrm{X}$ direction, the $\mathrm{Y}$ direction and the $\mathrm{Z}$ direction respectively.

Solving the two order differential equations (2) and substituting the obtained results into the equations (1), the corresponding amplitudes of vibration and arguments in the equations (2) can be obtained as:

$\left\{\begin{array}{l}\lambda_{y}=-\frac{2 m_{0} r \omega^{2}}{k-\omega^{2}\left(m+m_{0}\right)} \\ \lambda_{x}=-\frac{2 m_{0} r}{m+m_{0}} \\ \lambda_{\phi x}=-\frac{m_{0} r\left(L_{o z_{1}}-L_{o z_{2}}\right)}{J_{1}+J_{x}} \\ \lambda_{\phi y}=-\frac{m_{0} r\left(L_{o z_{1}}-L_{o z_{2}}\right)}{J_{2}+J_{y}} \\ \lambda_{\phi z}=-\frac{m_{0} r L_{o y}}{J_{3}+J_{z}}\end{array}\right.$ 

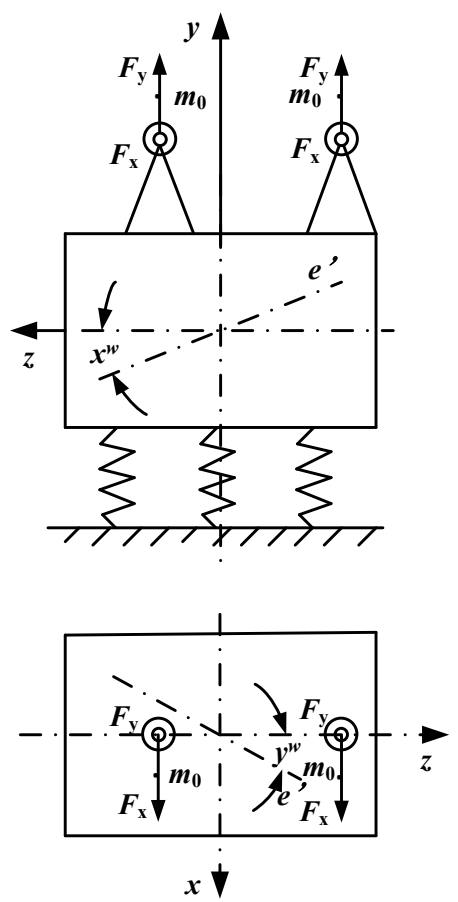

Fig. (3). The vibration mechanics model of the cropping machine.

Therefore, the equations of motion of any point in the vibration body can be written as:

$$
\left\{\begin{array}{l}
\mathbf{y}_{e}=y_{0}-\phi_{x} L_{e z}-\phi_{z} L_{e x} \\
\mathbf{x}_{\mathbf{e}}=x_{0}+\phi_{z} L_{e y}-\phi_{y} L_{e z} \\
\mathbf{z}_{\mathbf{e}}=\phi_{y} L_{e x}+\phi_{x} L_{e y}
\end{array}\right.
$$

when $m_{0}=3.7 \mathrm{~kg}, r=24.17 \mathrm{~mm}, m=248.3 \mathrm{~kg}, J_{\mathrm{x}}=0.258 \mathrm{~kg} \cdot \mathrm{m}^{2}$, $J_{\mathrm{y}}=0.915 \mathrm{~kg} \cdot \mathrm{m}^{2}, J_{\mathrm{z}}=0.657 \mathrm{~kg} \cdot \mathrm{m}^{2}, L_{\mathrm{oz} 1}=268.5 \mathrm{~mm}, L_{\mathrm{oz} 2}=296.4 \mathrm{~mm}$, $L_{\text {oy }}=177.0 \mathrm{~mm}$, the motion curves of the vibration body in all directions are shown in Fig. (4). As is shown in Fig. (4), the displacements of the vibration body in the $\mathrm{X}$ direction and the Y direction are basically the same. Therefore, the influence of the spring rigidity on the response of the vibration mechanism in the $\mathrm{X}$ direction and the $\mathrm{Y}$ direction is very little. However, the rotations of the vibration body around each axis are very different. The angles of rotation around the $\mathrm{X}$ axis and the $\mathrm{Y}$ axis are very little, and their differences are also small. The angle of rotation around the $\mathrm{Z}$ axis is about 5 times to that of rotation around the $\mathrm{X}$ axis. Therefore, in the course of the low-stress cropping, some necessary measures should be taken to reduce the influence on the cross-section quality. For example, the reasonable clamping position of the metal bar should be determined [9], and the structure of the eccentric block is changed into the adjustable eccentric block $[10,11]$ and the external force load mode is studied in detail.

\subsection{The Amplitude Frequency Response Characteristic}

As is shown in Eq. (3), there is an important relationship between the vibration amplitude of the vibration body in the $\mathrm{Y}$ direction and the excited frequency. Based on Eqs. (3)-(4),

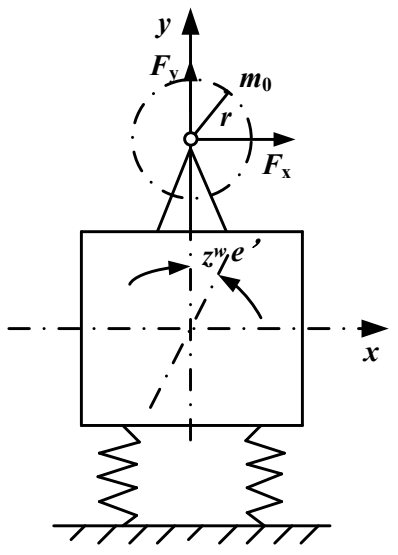

the amplitude frequency response characteristic curve of the vibration body in the $\mathrm{Y}$ direction is shown in Fig. (5). As is shown in Fig. (5), the vibration body in the $\mathrm{Y}$ direction has an obvious resonance region, and the resonance frequency is about $7.94 \mathrm{rad} / \mathrm{s}$. The amplitude of vibration of the vibration body within the existing excited frequency is increased significantly near the resonance region. Therefore, from the stability and reliability of the cropping machine in the course of the cropping, the cropping machine should be avoided working at this resonance frequency. However, in order to reduce the initiation time of crack, the machine is required to work at the resonance frequency of the vibration body in the crack initiation stage to provide greater force and amplitude, which is very helpful to realize the crack initiation of $\mathrm{V}$ shaped notch.

\section{THE EXPERIMENTAL RESULTS OF THE CROP- PING MACHINE}

When the diameter of the metal bar is $15 \mathrm{~mm}$, the radius at the $\mathrm{V}$-shaped groove tip is $0.2 \mathrm{~mm}$, the groove depth is $0.6 \mathrm{~mm}$, and the flare angle of $\mathrm{V}$-shaped notch is $90^{\circ}$, the cropping experimental results for 45 steel bars and 20 steel bars are shown in Figs. (6 and 7) respectively.

Due to the resonance effect, the initiation time of crack of $\mathrm{V}$-shaped notch tip is reduced remarkably. The cropping time for every segment of 45 steel bar and every segment of 20 steel bar is respectively about $18 \mathrm{~s}$ and $15 \mathrm{~s}$, which is also obviously less than the cropping time in [12]. According to the quality assessment parameters of cropping cross-section in [12], the maximum widths of the fault region (arrow area) in Fig. (6 and 7) are respectively $0.96 \mathrm{~mm}$ and $0.18 \mathrm{~mm}$, which is less than $1 \mathrm{~mm}$ and $0.2 \mathrm{~mm}$ in [12] under the same working conditions. 

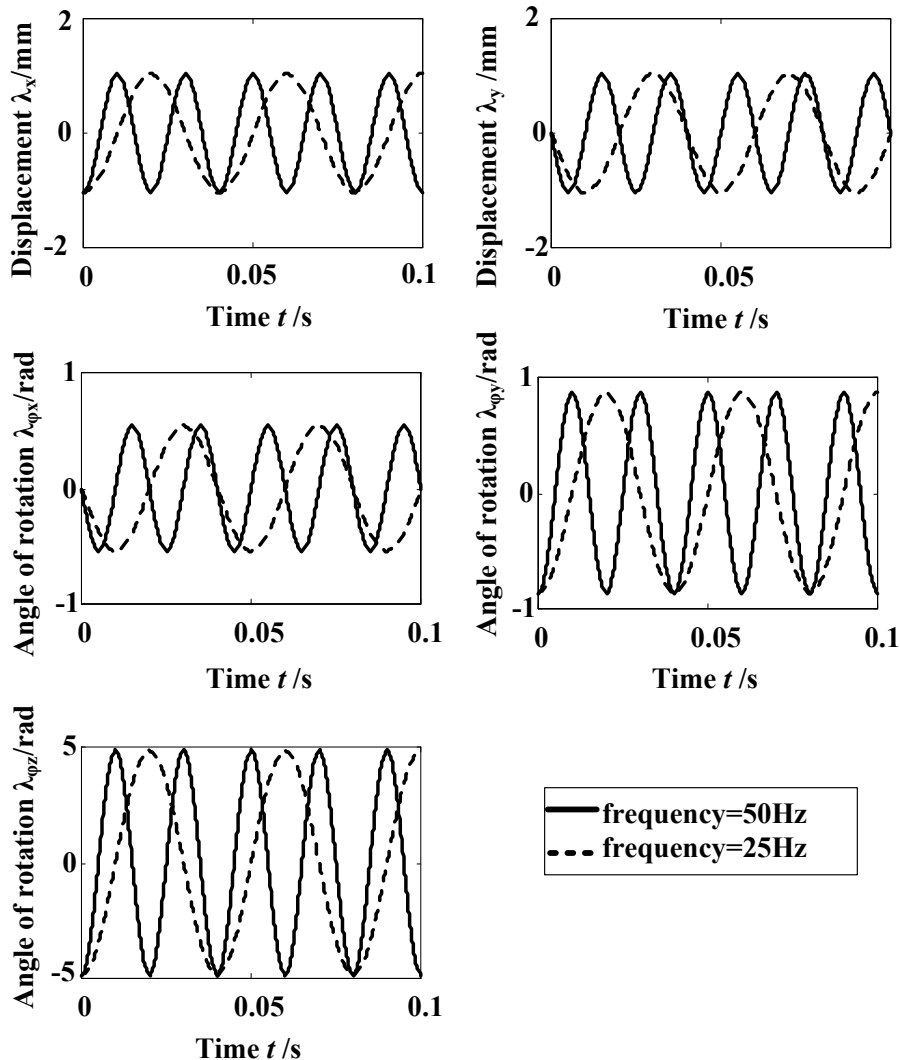

Fig. (4). The motion curves of the vibration body in all directions.

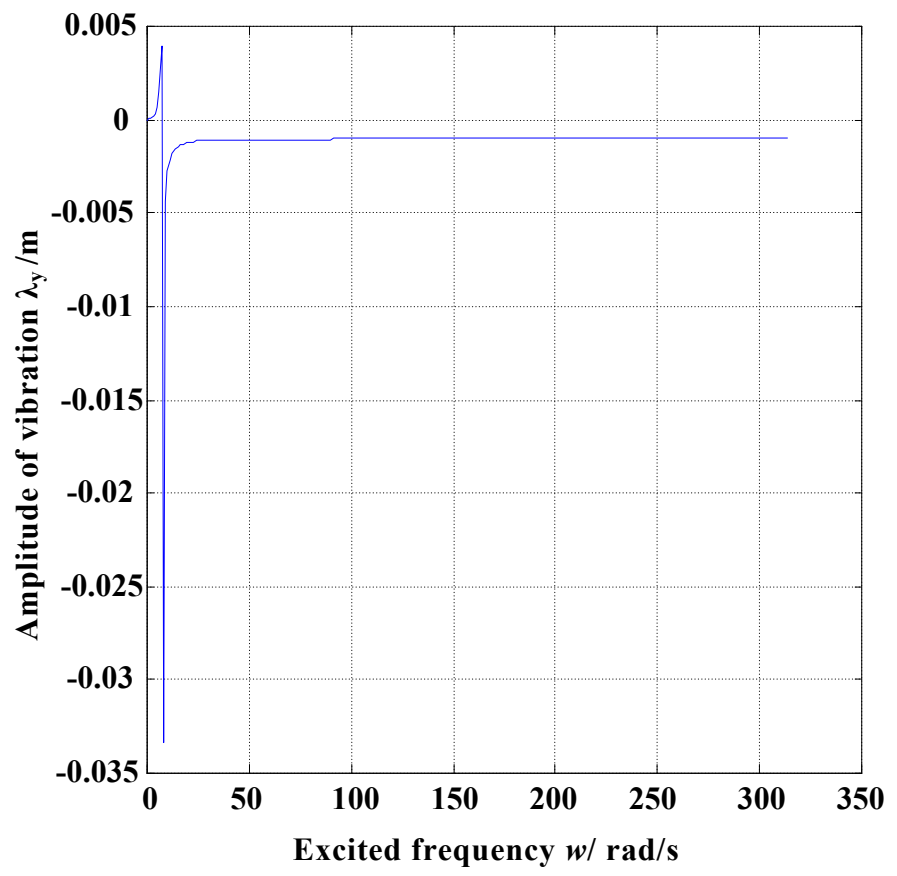

Fig. (5). The characteristic curve of amplitude frequency response.

The fatigue crack expands along the upper and lower direction of the bar cross-section of the V-shaped groove tip, which is mainly related with the force $F_{\mathrm{y}}$ in the $\mathrm{Y}$ direction acted on the bar, but the force $F_{\mathrm{x}}$ in the $\mathrm{X}$ direction acted on the bar is less than $F_{\mathrm{y}}$. It is also found in the cropping that the smaller the transient fault area is, the longer the cropping time is. 


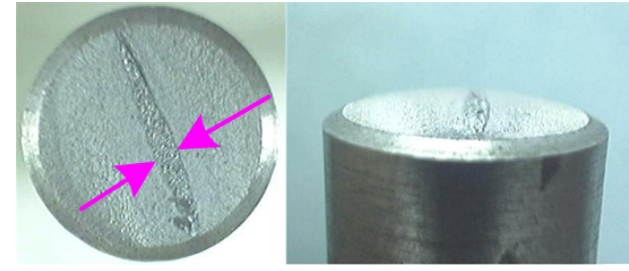

Fig. (6). The cross-section for 45 steel bar.

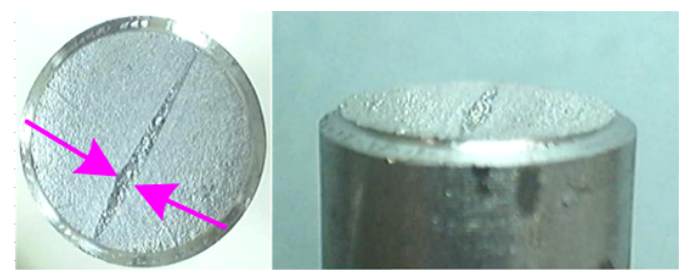

Fig. (7). The cross-section for 20 steel bar.

\section{CONCLUSION}

(1) Based on the effect of the stress concentration and the resonance effect, a new type of low-stress cropping method is proposed in this paper. The new type of cropping machine mainly consists of the electric machine, the eccentric block and the vibration body.

(2) The displacements of the vibration body in the $X$ direction and the $\mathrm{Y}$ direction are very small, and the angle of rotation of vibration body around the $Z$ axis is about 5 times that of rotation around the $\mathrm{X}$ axis or the $\mathrm{Y}$ axis. The vibration cropping machine in the $\mathrm{Y}$ direction has an obvious resonance region. To reduce the initiation time of crack, the machine is required to work at the resonance frequency of the vibration body in the crack initiation stage.

(3) The cropping experimental results show that the crosssections for 45 steel bars and 20 steel bars are very smooth and the cropping force acted on the metal bar is also reduced obviously. The smaller the transient fault area is, the longer the cropping time is.

\section{CONFLICT OF INTEREST}

The authors confirm that this article content has no conflict of interest.

\section{ACKNOWLEDGEMENTS}

The authors wish to express gratitude to the National Natural Science Foundation of PR China (Approval no. 51105382), Shandong Natural Science Foundation of PR China (Approval no. ZR2011EL039) and Qingdao Development Zone Science and Technology Development Project (Approval no. 2011-2-50), for supporting this work.

\section{REFERENCES}

[1] H.C. Gu, J.W. He, Save metal material manual. Machinery Industry Press: Beijing, 1995, pp. 2-10.

[2] J.D. Chen, Y.W. Wang, D.H. Yu, and Zi-gong Zhang, "Brittle precision cropping of metal materials", International Journal of Machine Tools and Manufacture, vol. 32, pp. 415-424, 1992.

[3] Y.F. Zhou, Intelligent Control for the system of frequency conversion and vibration about the low stress precision-shear. Master's degree thesis of Xi' an Jiaotong University, 2002.

[4] T. Song, Investigation in mechanism and control of a new type of precision cropping system with variable-frequency vibration method. Master's degree thesis of Xi' an Jiaotong University, 2004.

[5] H.X. Li, Virtual design of rotating and bending fracture machine. Master's degree thesis of Lanzhou University of Technology, 2007.

[6] Y. Tang, S.D. Zhao, and Z.W. Wang, "Experimental study on new precision cropping process for metal bars", China Mechanical Engineering, vol. 21, pp. 359-363, 2010.

[7] Y. Tang, S.D. Zhao, and J. Lin, "Experimental investigation of the effect of the circumferential loading of a rotary striking action cropping system", Journal of Engineering Manufacture, vol. 224, pp. 1095-1101, 2010.

[8] J.F. Li, Theoretical mechanics. Tsinghua University Press: Beijing, 2006, pp. 20-50.

[9] L.J. Zhang, S.D. Zhao, J. Lei, and W. Liu, "Investigation on the bar clamping position of a new type of precision cropping system with variable frequency vibration", International Journal of Machine Tools and Manufacture, vol. 47, pp. 1125-1131, 2007.

[10] L.J. Zhang, Y.R. Zhao, and S.D. Zhao, "Research on detection of new-type cropping machine based on LabVIEW", Manufacturing Technology \& Machine Tool, vol. 10, pp. 104-107, 2007.

[11] Han Xing, and Tao Jing, "Optimal structural design of complicated mechanical products based on data ming and muti-level partitioning strategy", International Journal of Applied Mathematics and Statistics, vol. 45, pp. 376-385, 2013.

[12] C.J. Hua, S.D. Zhao, L.J. Zhang, and W. Liu, "Investigation of a new-type precision cropping system with variable-frequency vibration”, International Journal of Mechanical Sciences, vol. 48, pp. 1333-1340, 2006. 